\title{
THE STATES ASSOCIATED WITH APPROXIMATELY INNER AUTOMORPHISMS
}

\author{
MARIE CHODA
}

\begin{abstract}
Let $M$ be a $\mathrm{II}_{1}$-factor acting standardly on a Hilbert space $\boldsymbol{H}$. For an approximately inner automorphism $\theta$ of $M$, there exists a state $\varphi$ on $B(H)$ associated with $\theta$. If the symmetry $\sigma$ of $M \otimes M$ is approximately inner on $M \otimes M$, then, by restricting the state associated with $\sigma$ to $B(H) \otimes I$, we have a hypertrace of $\boldsymbol{M}$.
\end{abstract}

1. Introduction. Let $M$ be a factor of type $\mathrm{II}_{1}$ with the canonical trace $\tau$, acting standardly in the Hilbert space $H=L^{2}(M, \tau)$. In [1], A. Connes proved that the following three statements are equivalent; (1) $M$ is hyperfinite, (2) the automorphism $\sigma$ on $M \otimes M$ (which is called the symmetry [2]) such that $\sigma(x \otimes y)=y \otimes x$ $(x \in M, y \in M)$ is approximately inner on $M \otimes M$, and (3) there exists a state $\varphi$ on $B(H)$ (which is called a hypertrace of $M)$ such that $\varphi(a x)=\varphi(x a)(x \in B(H)$, $a \in M)$. Following the order of $(1) \Rightarrow(3) \Rightarrow(2) \Rightarrow(1)$, he showed this result by passing through several other properties. As a consequence, if the symmetry $\sigma$ is approximately inner on $M \otimes M$, then there exists a hypertrace $\varphi$ of $M$, but it is not clear the relation between the symmetry $\sigma$ and the hypertrace $\varphi$.

In this paper, we shall show that, for an approximately inner automorphism $\theta$ on $M$, there exists a state $\varphi$ on $B(H)$ associated with $\theta$. And we shall show that if the symmetry $\sigma$ is approximately inner on $M \otimes M$, then the restriction of a state associated with $\sigma$ to $B(H) \otimes I$ is a hypertrace of $M$.

2. Results. Denote by $\zeta$ the canonical imbedding of $M$ into $H$ and by $J$ the canonical involution of the cyclic and separating vector $\zeta(1)$.

THEOREM 1. Let $\theta$ be an approximately inner automorphism of $M$; then there exists a state $\varphi$ on $B(H)$ such that $\varphi(\theta(u) J u J)=1$ for every unitary operator $u$ in $M$.

Proof. Since $\theta$ is approximately inner on $M$, by [1, Theorem 3.1], we have a bounded sequence $\left(x_{n}\right)_{n \in N}$ in $M$, not converging strongly to 0 , such that $x_{n} a-$ $\theta(a) x_{n}$ converges to 0 strongly for any $a$ in $M$. Hence, there exists a positive number $\alpha$ and a subsequence $\left(y_{n}\right)_{n \in N}$ of the sequence $\left(x_{n}\right)_{n \in N}$ such that $\left\|y_{n}\right\|_{2}>\alpha$. For each integer $n$, put $\xi_{n}=\zeta\left(y_{n}\right) /\left\|y_{n}\right\|_{2}$ and let $w_{n}$ be the vector state on $B(H)$ defined by $\xi_{n}$. Take a weak limit point $\varphi$ of the $\operatorname{set}\left\{w_{n} ; n=1,2, \ldots\right\}$. Then $\varphi$ is a desired state on $B(H)$, because, for every unitary $u \in M$, we have that $\left\|\xi_{n}-J u J \theta(u) \xi_{n}\right\|=\left\|y_{n} u-\theta(u) y_{n}\right\|_{2} /\left\|y_{n}\right\|_{2}$.

Received by the editors November 20, 1979.

AMS (MOS) subject classifications (1970). Primary 46L10.

Key words and phrases. $\mathrm{II}_{1}$-factor, trace, state, involution.

(C) 1981 American Mathematical Society 0002-9939/81/0000-0092/\$01.50 
We shall call $\varphi$ in Theorem 1 a state associated with $\theta$. A state $\varphi$ associated with an approximately inner automorphism $\theta$ is not necessarily unique for $\theta$ in general. But if $\theta$ is inner on $M$, then $\varphi$ is unique in the sense of the following. If $\theta$ is an inner automorphism on $M$ induced by a unitary $u$ in $M$, then we can take $\varphi$ as the vector state defined by $u$. Conversely if $\varphi$ is the vector state defined by a unitary $u$ in $M$, then $\theta$ is an inner automorphism induced by $u$. Specifically, $\theta$ is the identity on $M$ if and only if $\varphi$ is the trivial state (i.e. the vector state defined by the identity operator). In fact, if $\theta$ is inner on $M$, then by the proof of Theorem $1, \varphi$ becomes such as the state as above. Conversely, if there exists a unitary $v$ in $M$ such that $\varphi(x)=(x \zeta(v), \zeta(v))$ for every $x$ in $B(H)$, then for every unitary $u$ in $M$, we have that

$$
\left\|\theta(u) v u^{*}-v\right\|_{2}^{2}=\left(\zeta\left(\theta(u) v u^{*}-v\right), \quad \zeta\left(\theta(u) v u^{*}-v\right)\right)=2-2 \varphi(\theta(u) J u J)=0 .
$$

Since $\theta$ is an automorphism of $M$, it follows that $\theta(a)=v a v^{*}$ for all $a \in M$, so that $\theta$ is an inner automorphism induced by $v$.

COROLlaRY 2. Let $\varphi$ be a state associated with an approximately inner automorphism $\theta$ of $M$; then $\varphi$ is an extension of the canonical trace $\tau$ of $M$.

Proof. By Schwartz's inequality and the property of $\varphi$, we have that

$$
\varphi(J u J \theta(u) x)=\varphi(x)=\varphi(x J u J \theta(u)) \quad(x \in B(H), \text { unitary } u \in M) .
$$

Hence, $\varphi(a)=\varphi\left(\theta(u) a \theta\left(u^{*}\right)\right)$ for all $a$ in $M$ and all unitary $u$ in $M$. Since $\theta$ is an automorphism of $M$, then the restriction of $\varphi$ to $M$ is the canonical trace of $M$.

COROllary 3. If the symmetry $\sigma$ of $M \otimes M$ is approximately inner on $M \otimes M$, then a state $\varphi$ associated with $\sigma$ is an extension of a hypertrace of $M$.

Proof. By the equality (*), for all $x$ in $B(H)$ and all unitary $u$ in $M$, we have that

$$
\begin{aligned}
\varphi(u x \otimes 1) & =\varphi\left((1 \otimes J u J)(u \otimes 1)\left(x \otimes J u^{*} J\right)\right) \\
& =\varphi\left(\left(x \otimes J u^{*} J\right)(1 \otimes J u J)(u \otimes 1)\right)=\varphi(x u \otimes 1),
\end{aligned}
$$

so that, $B(H) \ni x \rightarrow \varphi(x \otimes 1)$ is a hypertrace of $M$.

\section{REFERENCES}

1. A. Connes, Classification of injective factors, Ann. of Math. (2) 104 (1976), 73-115.

2. S. Sakai, Automorphisms and tensor products of operator algebras, Amer. J. Math. 97 (1975), 889-896.

Department of Mathematics, Osaka Kyotku University, Tennoj, Osaka 543, Japan 